\title{
Current state of imaging in dermatology
}

\author{
Brian P Hibler, BS; Qiaochu Qi, BA, MPH; and Anthony M Rossi, MD
}

\section{Abstract}

Medical imaging has dramatically transformed the practice of medicine, especially the field of dermatology. Imaging is used to facilitate the transfer of information between providers, document cutaneous disease, assess response to therapy, and plays a crucial role in monitoring and diagnosing skin cancer. Advancements in imaging technology and overall improved quality of imaging have augmented the utility of photography. We provide an overview of current imaging technologies used in dermatology with a focus on their role in skin cancer diagnosis. Future technologies include three-dimensional, total-body photography, mobile smartphone applications, and computerassisted diagnostic devices. With these advancements, we are better equipped to capture and monitor skin conditions longitudinally and achieve improved diagnostic accuracy of skin cancer.

Semin Cutan Med Surg 35:2-8 @ 2016 Frontline Medical Communications

$\mathrm{M}$ edical imaging has dramatically transformed how clinicians evaluate, diagnose, monitor, and treat disease. The highly visual nature of cutaneous diseases makes digital imaging extremely useful in everyday practice. Imaging facilitates the exchange of information between physicians, as well as with patients. Photography makes it possible to document and transmit a large amount of clinical information in a single image, and a number of technological advancements have improved our ability to digitally image and record clinical findings. One critical application of these technologies is for the early, more accurate diagnosis of skin cancer. We will discuss the role of imaging in dermatology, review current and future technologies, and examine the challenges that exist which must be overcome to advance imaging in dermatology with a focus on improving skin cancer diagnosis and management.

\section{Imaging in dermatology}

\section{Facilitating communication}

Dermatologists have developed very specific, detailed terminology to paint a vivid picture that other clinicians may use to visualize exactly how the cutaneous findings presented. The physical examination of skin conditions relies on subjective assessment, and

Memorial Sloan Kettering Cancer Center, Department of Dermatology, New York, New York.

Disclosures: Mr Hibler, Ms Qi, and Dr Rossi have nothing to disclose. Correspondence: Anthony M. Rossi, MD; Memorial Sloan Kettering Cancer Center; 4th Floor Dermatology; 16 E. 60th Street, New York, NY 10022. Email: anthonymrossi@gmail.com. descriptions of the same lesion may vary among providers. Furthermore, clinicians who are not as well versed in the terminology of dermatologists may not be able to fully appreciate the illustrative language in written text. Standard digital photography, which may be captured and uploaded to the patient's chart in seconds, overcomes these limitations of written text and can be used to show the skin exam of the entire body or may be limited to individual lesions.

\section{Documenting cutaneous conditions}

Many dermatologists use imaging in their everyday practice to document the presentation of cutaneous disease. Images may be used to compare disease states before and after treatment to evaluate efficacy, recall precise location of lesions after biopsy or excision, and/or monitor disease progression over time. Imaging captures the exact morphology of cutaneous findings and provides visualization of subtle details. More specialized technologies can be used to view cellular structures below the surface of the skin, enhancing diagnostic accuracy. Imaging is extremely valuable for both benign and malignant lesions, and plays an important role in professional communications, practice development, and resolving medicolegal issues. ${ }^{1}$

\section{Imaging in skin cancer}

Imaging in dermatology has come to play a critical role in the evaluation and monitoring of skin cancer. Although clinicians primarily rely on their eyes, a number of instruments have been developed to improve melanoma detection. For pigmented lesions, the ABCDE criteria is an effective screening tool for melanoma. ${ }^{2}$ The importance of a changing lesion (evolution [E]) was described in 2004 by Abbasi et al, underscoring the importance of accurately documenting changes in pigmented lesions. ${ }^{3}$ The number needed to biopsy to capture a melanoma may be as high as 80:1 in certain populations, highlighting the need for improved imaging tools to identify and monitor suspicious lesions. ${ }^{4}$ Imaging may be at the level of total body photography to detect changes in size, shape or color of individual lesions, or the subcellular level with reflectance confocal microscopy to visualize atypical cells. Photography is used to identify specific lesions within a field of similar-appearing lesions, monitor at-risk patients for changing lesions, and can ultimately assist in guiding biopsies. It is also useful for identifying sites of prior excisions, improving our ability to monitor for recurrence.

\section{Current technologies (Table) Digital photography}

Digital photography is widely used by dermatologists. A recent survey to board-certified practicing US dermatologists showed that $82.2 \%$ use digital photography. ${ }^{5}$ Younger dermatologists (under 40 years old) and those working at academic settings use digital pho- 


\section{TABLE. Current imaging modalities in dermatology}

Technique
Digital photography,
Total body photography
(TBP),
UV photography
Dermoscopy

Reflectance confocal microscopy (RCM)

\section{Advantages} management. and skin self-examination. changes, especially with TBP. visualization of body surface. an educational tool regarding sun protection. of skin lesions. correlates well with histopathologic features. compared to unaided eyes.
Inexpensive, allows for long-term data storage, easy

Facilitates communication, documentation, education,

Long term monitoring of patient to detect any suspicious

3D-TBP generates a 3D avatar, allowing for enhanced

UV photography can assess sun damage and serves as

Magnifies skin 10x to facilitate diagnosis of a wide range

Monitors skin lesions over time to detect subtle changes.

Established criteria for diagnosing skin cancer that

Diagnoses pigmented and non-pigmented skin cancer with improved sensitivity, specificity, and accuracy

High resolution equivalent to $30 \mathrm{X}$ on high magnification histopathology, which allows for imaging of microscopic structures.

Allows for imaging to a depth of $200 \mu \mathrm{m}$ down to papillary dermis.

Noninvasive and may reduce the need for biopsy.

Low power laser does not damage tissue.

Facilitates diagnoses of equivocal features, allows for delineation of surgical margins, and useful for long-term monitoring.

Optical coherence tomography
Noninvasive and may reduce the need for biopsy.

High resolution of 3-15 $\mu \mathrm{m}$ allows for imaging of microscopic features.

Depth of $1.5 \mathrm{~mm}$ is better than RCM.

Generates 2D and 3D images.

Wide applications for imaging lesions, aging skin, skin moisture, and engineered tissues.

Can be combined with other techniques including Doppler to enhance diagnostic accuracy.

\section{Limitations}

Only captures skin surface morphology.

Traditional 2D-TBP is time consuming and may be uncomfortable to patient.

Requires consent and secure handling and storage of photos to ensure patient privacy.

Proper training is needed.

Interpretation of results is subjective.

Limited magnification restricts its applications.

Anchoring bias and search satisfaction can limit diagnostic accuracy.

Requires proper training and has an associated learning curve.

Unable to image lesions beyond papillary dermis, thus cannot reliably evaluate tumor invasion.

Expensive and requires proper training and experiences

Strong scattering limits the depth to thin tumors and cannot reliably image deeper tumor invasion.

Cannot differentiate between benign and malignant lesions effectively due to limited resolution. tography more often, at $90.1 \%$ and $93.9 \%$, respectively. ${ }^{5}$ Overview and close-up photos can be taken at baseline and follow-up visits to track lesions over time. Especially in the case of melanoma, early detection is crucial and can significantly reduce mortality. In one study, $44 \%$ of melanoma was detected in situ with a lower thickness in the group with baseline photography, compared to $35 \%$ for the general population. ${ }^{6}$ Another study showed that baseline photography aids early detection of melanoma in long-term follow-up of high-risk patients. ${ }^{7}$

Total-body photography (TBP) is a type of digital photography used to globally survey existing lesions and to identify new or changing lesions. In traditional TBP, the photographer (physician, nurse, or technician) takes an average 24 photos (range, 4-50) of the patient in various positions. ${ }^{8}$ Close-up photos may be taken for selected lesions, and photos are taken at regular intervals for long- term follow-up. TBP has been shown to help detect early melanoma. ${ }^{9}$ The addition of TBP to skin self-examination (SSE) increases the sensitivity for detecting new or changed nevi from $60 \%$ to $72 \%$ and increases specificity from $96 \%$ to $98 \% .{ }^{10}$ Patients also tend to be more compliant with SSE when provided with TBP. ${ }^{10}$ One limitation of traditional TBP is that it is a time consuming procedure requiring patients to be exposed for a relatively long period of time. Patients also must be photographed in a variety of positions to capture all surfaces of the body.

\section{Dermoscopy}

Dermoscopy is used to visualize subsurface structures in the skin that cannot be detected by the naked eye. The dermatoscope is a hand-held, optical device with 10x magnification and a transilluminating light source. Applications of dermoscopy include evalu- 
ating inflammation, autoimmune diseases, infection, and hair and scalp disorders. Polarized dermoscopy provides better visualization of the structures common in skin cancer, including vascular and crystalline structures. ${ }^{11}$ Dermoscopic photographs can be captured, allowing for enhanced viewing of suspicious lesions and for monitoring subtle changes over time.

Studies have found dermoscopy improves the sensitivity and accuracy of detecting melanoma compared to unaided eyes. A recent meta-analysis of 9 prospective studies in clinical settings demonstrated that the relative diagnostic odds ratio for melanoma of dermoscopy compared to unaided eyes was 9.0 (95\% CI 1.5-54.6; $P$ $=.03$ ) after removal of 2 outlier studies. ${ }^{12}$ For 7 of the 9 studies, the positive predictive value was greater for dermoscopy than for the unaided eye, and dermoscopy provided an estimated $18 \%$ improvement in sensitivity $(95 \%$ CI $9-27 ; P=.002) .{ }^{12}$ Previous metaanalyses also showed significantly improved diagnostic accuracy for melanoma using dermoscopy compared to unaided eyes. ${ }^{13,14}$ Use of dermoscopy yields better specificity as demonstrated by the $42 \%$ reduction of patients referred to biopsy in a randomized trial ${ }^{15}$ and a reduced benign/malignant ratio of excised melanocytic lesions from $18: 1$ in predermoscopy era to $4: 1$ in post-dermoscopy era $(P=.04) .{ }^{16}$ Dermoscopy also improves accuracy of detecting non-melanocytic lesions, including basal cell carcinoma. ${ }^{17}$

In addition to diagnosing skin lesions, dermoscopy is used in monitoring as well. Sequential digital dermoscopy imaging (SDDI) is used to follow skin lesions and detect any suspicious change. Research has shown that SDDI allows for early detection of changes in melanomas that still lack dermoscopic evidence of melanoma. ${ }^{18}$ SDDI has also been shown to reduce unnecessary biopsies and improve accuracy when used with short-term clinical monitoring of melanocytic nevi at 3-month intervals. ${ }^{19}$ Smartphone attachments allow clinicians to readily acquire demoscopic images and upload them to the patients chart for expedient SDDI (Figure 1).

Although widely used, dermoscopy requires proper training. Accurate diagnosis is based on the physician's interpretation, and studies have shown dermoscopy by untrained or less experienced individuals was no better than inspection without dermoscopy. ${ }^{13}$ Its use should always be based on the clinical context or else accuracy is compromised. In addition, anchoring bias and search satisfaction can result in inaccurate diagnosis.

\section{Reflectance confocal microscopy}

Reflectance confocal microscopy (RCM) allows for noninvasive, high-resolution (30x) imaging of skin to a depth of $200 \mu \mathrm{m}$, reaching the papillary dermis. ${ }^{20}$ The light source is a low-power laser emitting near-infrared light, which scans across the sample (Figure 2). Light from a desired focal point is then reflected and passes through a pinhole and enters the detector. Different cells and structures have different reflection indexes, giving contrast to the image. Melanin and keratin have high contrasts with reflection indexes of 1.7 and 1.5 , respectively. ${ }^{20}$ High contrast structures appear bright/ white, and output images are horizontal sections of the skin.

Compared to histopathology, RCM preserves the natural structure of the tissue. Its high resolution enables imaging of nuclear, cellular, and tissue architecture, and its noninvasive nature avoids unnecessary biopsy. One study showed the number needed to excise a melanoma decreased from 14.6 to 6.8 using RCM. ${ }^{21}$ RCM can be a valuable adjunct tool to diagnose skin lesions with equivo- cal features. It is also used to delineate surgical margins and to monitor patient's response to non-surgical therapies. ${ }^{22-24} \mathrm{RCM}$ has been utilized to evaluate tumors in sensitive areas, such as the eyelid, ${ }^{25}$ and oral and genital mucosa. ${ }^{26}$ Algorithms are developed to aid detection of skin cancer with RCM, which has achieved high

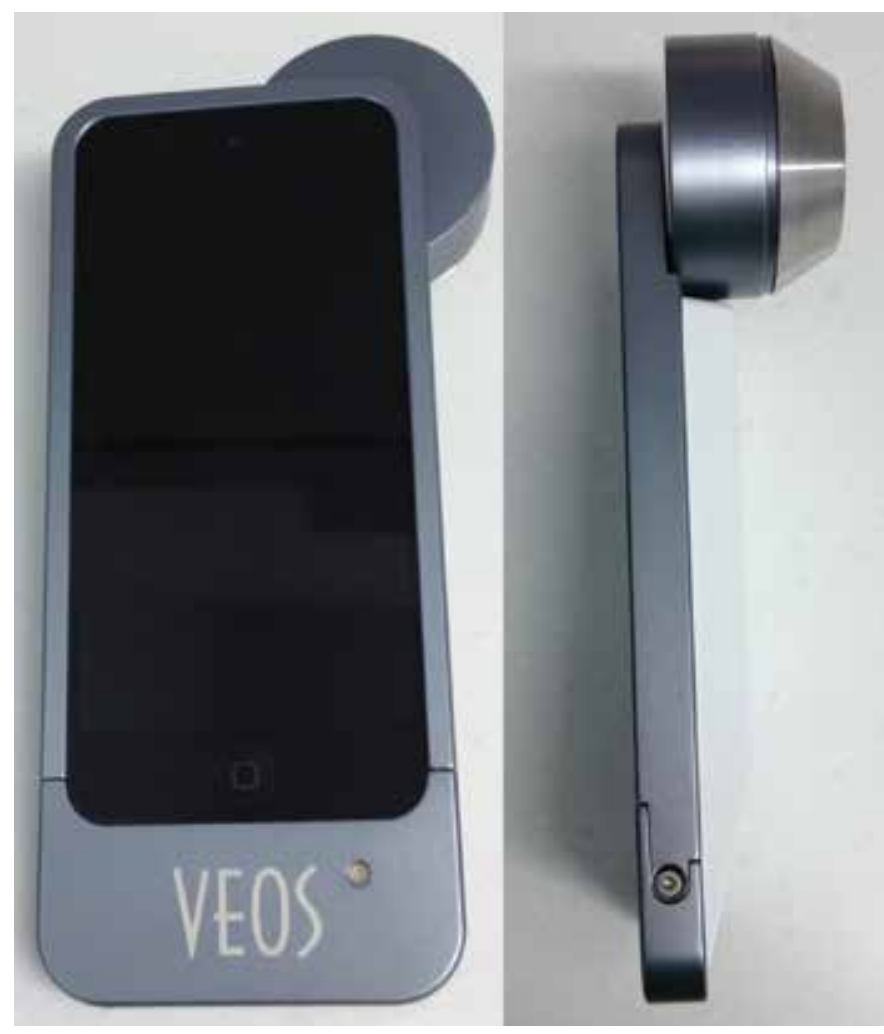

FIGURE 1. Mobile dermatoscope attachment allows for simultaneous demoscopic evaluation and image capture (Canfield Scientific Inc, Fairfield, NJ).

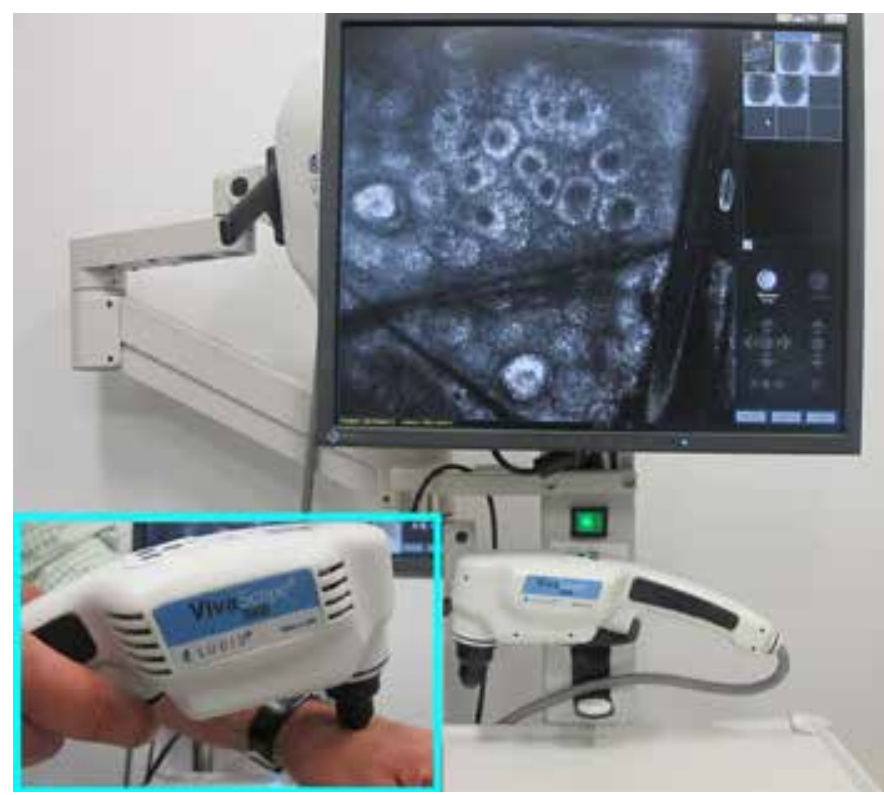

FIGURE 2. Vivascope Reflectance Confocal Microscope System (Lucid, Inc, Rochester, NY). Inset shows the handheld Vivascope 3000 . 


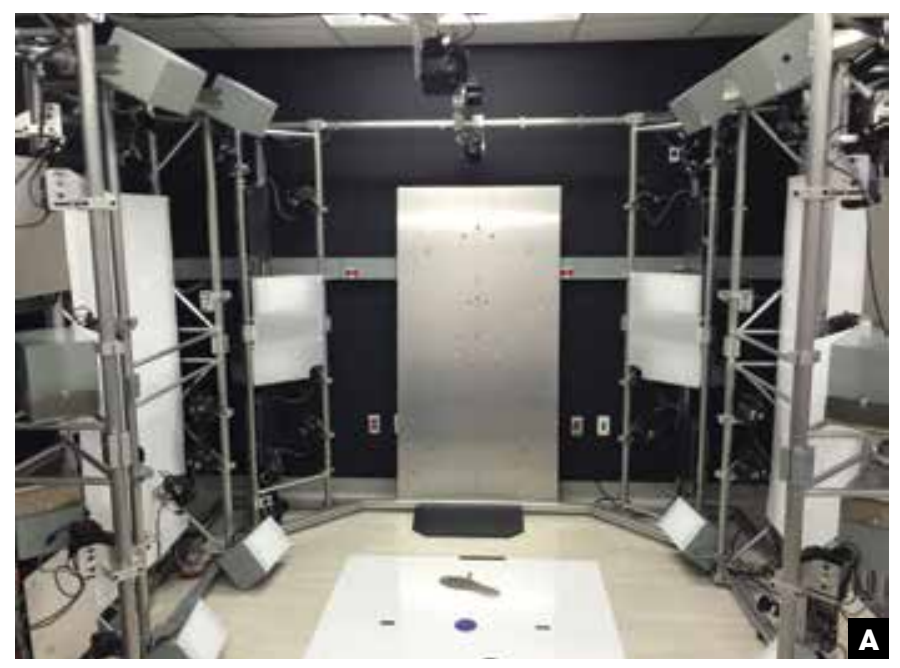

FIGURE 3. A) Three-dimensional total body photography apparatus (Canfield Scientific Inc, Fairfield, NJ). B) Representative 3D total body photography avatar with touchscreen interface allowing for enhanced viewing and demarcation of suspicious lesions. C) Avatar can be rotated 360 degrees to view all body surfaces.

accuracy. According to recent studies, sensitivity and specificity for diagnosis of skin cancer were $88.9 \%-96.5 \%$ and $79.3 \%-94.1$, respectively. ${ }^{27,28}$

RCM is a valuable imaging tool, and the advent of the smaller, more portable handheld RCM device allows for improved imaging of concave surfaces and difficult locations such as the corner of the eye. However, RCM also has limitations. It can only image to the depth of papillary dermis, and lesions in deeper layers of the dermis cannot be seen. Therefore, a negative result with RCM cannot rule out potential tumors in deep dermis. Furthermore, there is a learning curve associated with RCM imaging. Clinicians need formal training and experiences to properly use RCM. Yet, the training required for accurate RCM interpretation has been reported to be less than that of dermoscopy. ${ }^{29}$ Other practical limitations to widespread adoption of RCM at this time include its initial cost of the device and time required for imaging large or multiple lesions. ${ }^{30}$

\section{Optical coherence tomography}

Optical coherence tomography (OCT) is a noninvasive imaging technique using infrared light and operates analogously to ultrasound. It has a resolution of 3-15 $\mu \mathrm{m}$ at a depth of up to $1.5 \mathrm{~mm}^{31}$ and can generate real-time $2 \mathrm{D}$ and $3 \mathrm{D}$ images of the tissue. OCT is used to visualize skin morphology, including structures like hair follicles, blood vessels, glands, epidermis, dermo-epidermal junction, and dermis. ${ }^{31}$ It also aids the diagnosis of skin lesions including cancer and inflammatory diseases, and is used to monitor patient's response to treatment. Some other applications include assessment of skin moisture, burn depth, wound healing, skin atrophy, UV damage, and engineered tissues. ${ }^{32}$ Diagnostic criteria using OCT have been developed for skin tumors, and studies have shown promising results. One study using OCT to diagnose basal cell carcinoma showed the specificity and sensitivity to be $75.3 \%$ and $95.7 \%$; the overall accuracy was $87.4 \% .{ }^{33}$ All numbers were higher than using dermoscopy alone. The study also showed superior specificity for OCT compared to dermoscopy alone in di-
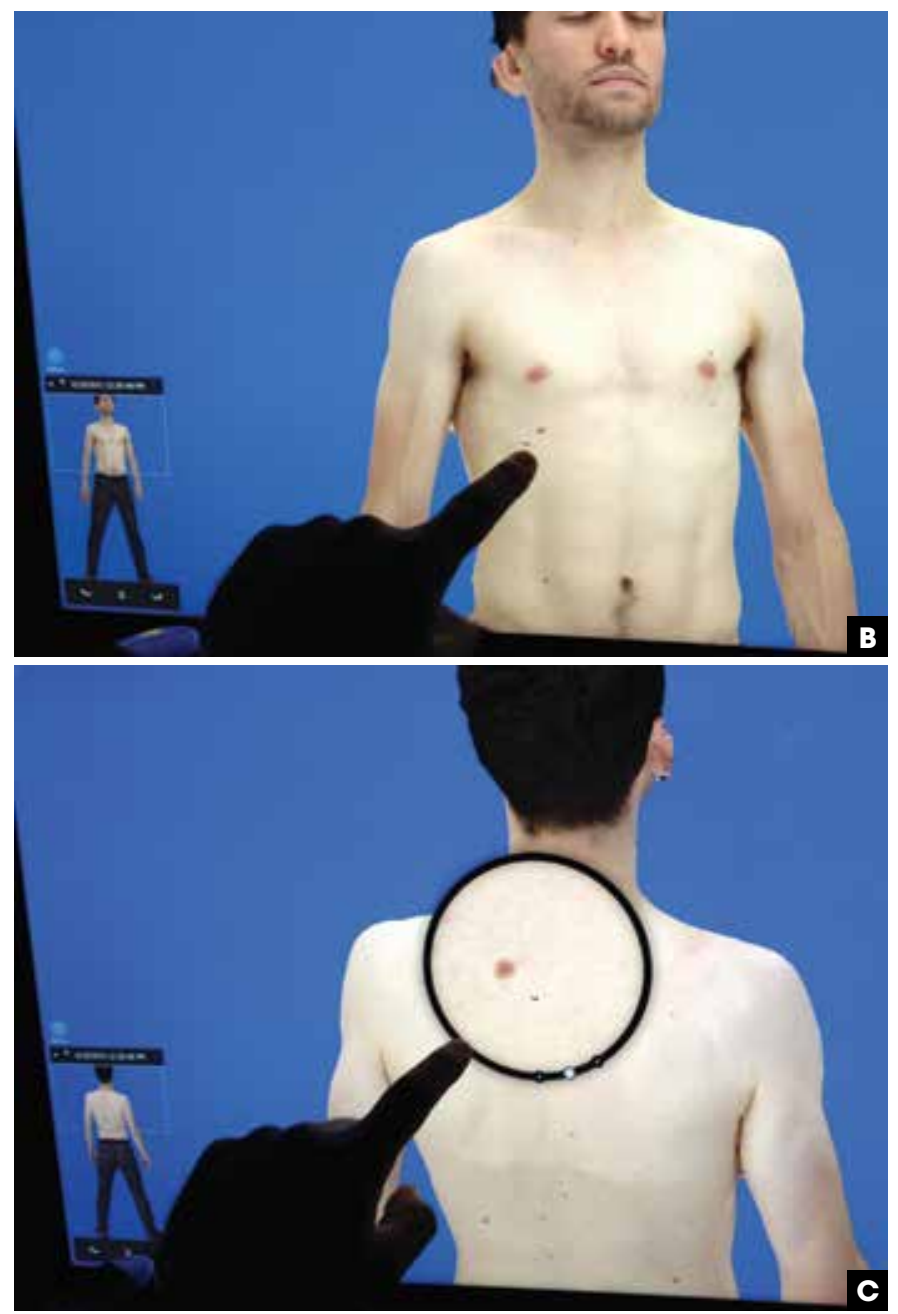

agnosing actinic keratosis, Bowen's disease, seborrheic keratosis, and inflammatory diseases.

Optical coherence tomography measures the depth of invasion better than conventional scanning laser microscopy. However, it still has a limited depth of $1.5 \mathrm{~mm}$ due to strong scattering and thus can only image relatively thin tumors. It also has limited resolution and cannot effectively differentiate between benign and malignant lesions. ${ }^{34}$ Research is still needed to evaluate the use of OCT in the diagnosis of melanocytic lesions. Current improvements include adopting Doppler techniques to measure velocity of blood flow and combining OCT with spectroscopy to enhance diagnostic accuracy. ${ }^{32}$

\section{Future technologies}

There are a number of innovative imaging devices on the horizon that aim to overcome limitations of existing modalities. These range from whole body photography, to putting imaging in the hands of the patient, to automated computer-assisted diagnostic modalities. Each of these technologies may play a unique role in assisting with earlier and more accurate diagnosis of skin cancers.

\section{3-D total-body photography}

Traditional total-body photography is experiencing an evolution from a series of 2-dimensional images to creating a 3-dimensional representation of the patient. It uses a series of 46 cameras that synchronously capture an image of the patient in three tenths of 
a second (Canfield Scientific Inc, Fairfield, New Jersey; Figure $3 \mathrm{~A})$. Within minutes, a computer program assembles the images to recreate a 3-dimensional digital model of the patient showing all skin lesions (Figure 3B). Individual lesions that wish to be closely monitored may be imaged with close-up photographs or dermoscopic photographs which are then tagged to the lesions on the digital model. This approach permits accurate registration of images with their exact locations on the individual and allows for analysis and monitoring by the dermatologist. Because change in a lesion is the most sensitive way to detect skin cancer early, it is hypothesized that this approach may allow for closer monitoring of suspicious lesions with more accurate assessments of change over time, reducing unnecessary biopsies and capturing malignant transformation at an earlier stage.

The 3-dimensional imaging approach offers a number of advantages over the standard 2-dimensional imaging technique. First and foremost, the time required to image the patient is significantly shorter, requiring only one near-instantaneous image capture rather than the repositioning and series of photographs required with the two-dimensional approach. This means less time for the patient to be exposed and only one pose necessary, creating an overall more comfortable experience. The images in the three-dimensional TBP are rendered into one comprehensive digital model, eliminating the overlap between adjacent images that occurs with 2-dimensional TBP. This helps create consistency in the appearance of the size of lesions, whereas with the 2-dimensional imaging, if an image is taken at an angle, it may appear larger than its actual size. Additionally, 3-dimensional imaging recreates the surface texture and allows a lesion to be assessed from all angles. The digital model can be moved and rotated as desired to gain a better visualization of a particular lesion (Figure 3C), and serial close-up dermoscopic photographs can be attached to individual lesions to provide even more details. This is particularly useful for viewing moles on curved body surfaces.

Although the 3-dimensional imaging approach offers a number of advantages over standard 2-dimensional TBP, there are limitations to this approach. Three-dimensional TBP is currently unable to accurately capture lesions on the scalp, the soles of feet, and within body folds. As such, these locations need to be independently assessed and documented with separate photography. Furthermore, studies comparing the efficacy of the $2 \mathrm{D}$ approach versus the $3 \mathrm{D}$ approach, as well as demonstrating the cost-effectiveness, have yet to be elucidated.

\section{Smartphone imaging and the mobile market}

Technological advancements in smartphone technology have augmented on-the-go imaging in a myriad of ways. The majority of physicians own and use a smartphone, and the ease with which one can obtain and readily share images using mobile photography makes it useful in the clinic. Previously, the image quality afforded by mobile phones limited its utility. However, image resolution has improved and may no longer be a significant limiting factor. Improvements in smartphone photography continue to have a profound impact on imaging by both patients and physicians.

The role of smartphone technology in the monitoring of lesions and diagnosis of skin cancer has yet to be well-studied. The results of a population-based survey of individuals with melanoma found that over half of all melanomas were self-detected. ${ }^{35}$ As such, it is likely that novel utilization of mobile technology may augment an individual's ability to detect melanoma using the built-in camera in combination with smartphone applications. A recent study evaluated the ability of a mobile smartphone application using novel fractal image analysis to guide diagnosis of pigmented lesions. The application had a sensitivity of $73 \%$ and a specificity of $88 \%$, which was comparable to that observed with clinical diagnosis (sensitivity $88 \%$, specificity $97 \%$ ). ${ }^{36}$ While this technology is still inferior to a clinical examination by a dermatologist, it suggests the possibility of future mobile smartphone applications.

It is difficult to identify which of the mobile applications are most reliable and accurate in terms of content, image registration, and image analysis. Until the diagnostic accuracy of these applications is better studied, the mobile market should be used for education and to capture images that may be utilized in a clinical setting. Photodocumentation of lesions using smartphone technology may help facilitate patient-doctor communication and may be best used to track changes in pigmented lesions or document biopsy or surgical sites. ${ }^{37}$ Nevertheless, regular physician examinations are required for complete skin examinations that may reveal lesions or skin cancers unnoticed by the patient.

\section{Machine-assisted diagnosis/multispectral imaging}

Diagnosing skin cancer based upon the clinical appearance of a lesion is highly subjective. The varied presentations of melanoma make it even more diagnostically challenging and underscore the need for additional clinical tools to increase diagnostic accuracy. Utilization of a computer-assisted algorithm may provide quantitative analysis of lesions, yielding less inter-physician variability. There is ongoing investigation into devices using automated analysis of digitized images as a way to improve diagnosis and reduce unnecessary biopsies.

There are two main multispectral imaging devices currently under investigation for their role in the improving detection of melanoma. The first is the SIAscope, using spectrophotometric intracutaneous analysis (SIA) to evaluate pigmented lesions. This noninvasive, multispectral imaging device uses wavelengths of light ranging from 400-1000 nm to produce eight narrow-band spectrally filtered images over areas of skin from $1.2-2.4 \mathrm{~cm}^{2}{ }^{38}$ The device quantitatively measures the amounts of certain chromophores in the skin, including hemoglobin, collagen, and melanin. Using this information regarding the vascularity of the skin, integrity of collagen, and presence of dermal pigment, the device attempts to diagnose skin cancer. Several studies have evaluated the use of SIAscopy versus dermoscopy for pigmented lesion analysis. Haniffa et al evaluated 881 pigmented lesions (31 melanomas) using dermoscopy followed by SIAscopy. ${ }^{39}$ The sensitivity and specificity using dermoscopy was $94 \%$ and $91 \%$, respectively, and was not improved by the addition of SIAscopy ( $87 \%$ and $91 \%$, respectively). In a prospective, double-blind study, Glud et al. evaluated lesions in which a diagnosis of melanoma could not be ruled out clinically by a nondermatologist using dermoscopy and SIAscopy ${ }^{40}$ Both devices overestimated the proportion of possible malignant lesions. The reported sensitivities were $92 \%$ and $100 \%$ and specificities were $81 \%$ and $59 \%$, respectively. Both studies concluded that, at the present time, dermoscopy remains the best diagnostic tool for preoperative evaluation of lesions suspicious for melanoma. However, newer versions of the SIAscope are capable of capturing dermoscopic images, making this useful for archiving 
images and training purposes. Furthermore, the addition of novel scoring algorithms (eg, MoleMate, MedX, Mississauga, Ontario, Canada), which build upon current SIA technology, may prove to be cost-effective in diagnosing and managing suspicious pigmented lesions. $^{41}$

The other diagnostic device is the MelaFind (MELA Science Inc, Irvington, New York), a hand-held, fully-automated lesion analysis system which uses pattern recognition for quantitative and objective monitoring of pigmented lesions over time. ${ }^{42}$ The device illuminates the skin using 10 different narrow spectral bands in the visible and near-infrared (wavelength 430-950 $\mathrm{nm}$ ) region, and compares acquired images to a database of known melanomas and benign lesions. The software determines the border of lesion and analyzes for asymmetry, coloration, change in perimeter, texture change, and wavelet maxima. Based upon this information the output is binary: "biopsy" or "no biopsy needed." Clinical studies showed that compared to dermatologists, Melafind had a higher sensitivity (96\%-98\%) but consistently lower specificity $(8 \%-44 \%) .{ }^{43-45}$ While the sensitivity of the device is high, it has been criticized for recommending a biopsy in up to $90 \%$ of lesions evaluated, calling into question its diagnostic utility. ${ }^{46}$ As a result, it is suggested that Melafind be used as an adjunct to the clinical exam by dermatologists. In studies by Rigel et al and Hauschild et al, dermatologists using Melafind had increased sensitivity (69\% to $94 \%$ and $70 \%$ to $78 \%$ ) with a modest decrease in specificity $(54 \%$ to $40 \%$ and $56 \%$ to $46 \%){ }^{47,48}$ These results suggest that Melafind may improve the final biopsy decision when used by dermatologists, as the risk of not biopsying a malignant lesion has far greater consequences than biopsying a nonmalignant lesion.

There are challenges that must be overcome for multispectral imaging to play a meaningful role in evaluating lesions. First and foremost is the diversity and complexity of clinical lesions. When considering just melanocytic lesions, there are a number of clinical entities that mimic melanoma. Moreover, melanoma may take on a number of different presentations, including amelanotic lesions or those less than the diagnostic cutoff of $6 \mathrm{~mm}$ diameter. Even now, once a lesion is excised, there is a reported discordance rate of $14 \%$ among pathologists for melanoma diagnosis at a leading melanoma referral center. ${ }^{49}$ As such, automated diagnostic devices must take into account the myriad of clinical presentations and need to be tested in a large set of biopsy-proven lesions. Furthermore, the different backgrounds of skin (color, degree of photodamage, etc) and the context of overall skin condition need to be considered. For this reason, it is still up to the clinician to select which lesions to evaluate. Currently, emphasis has been given to automated diagnosis of melanoma due to its associated mortality compared with nonmelanoma skin cancers. However, it is likely that for widespread clinical adoption, such a device will need to be capable of characterizing both melanoma and nonmelanoma skin cancers. As such, multimodal spectral diagnostic devices are under investigation for their ability to diagnose both melanoma and nonmelanoma skin cancer. ${ }^{50}$

\section{Challenges}

Advanced imaging technology has drastically improved patient care in dermatology. At the same time, challenges remain. Cost is one concern when choosing an imaging modality. In a survey to board certified US dermatologists, $18 \%$ did not adopt digital photography in clinical practice, with the majority citing cost as the prohibiting factor. ${ }^{5}$ More advanced technologies, including 3DTBP, RCM, and OCT require complex machinery and computer systems, which harbor significant upfront costs. At the same time, training is required for clinicians to use advanced imaging technology properly, further increasing cost and limiting widespread adoption. Lastly, a lack of standardization of imaging technique may lead to variable image quality and prohibit the exchange of images between providers. ${ }^{51}$

Another concern is patient privacy, especially with digital photography and mobile technology. It is difficult to de-identify photographs. Masking, such as pixelating or putting a black bar over the eyes, is used to conceal identity. More recently, the standard is to crop or remove identifying features carefully. Clinicians must be careful and follow proper consent procedures before taking and disseminating photos of patients, whether for publication or for communicating with other physicians. Mobile technology such as smartphones facilitates communication and is convenient, but raises issues with patient privacy and confidentiality. In a recent survey of patient viewpoints, $65.5 \%$ of patients expressed concern regarding confidentiality, and the majority $(97.7 \%)$ preferred their pictures be taken with a hospital-owned camera to a smartphone. ${ }^{52}$ Care must be taken to balance the convenience of mobile technology with patient comfort and confidentiality. ${ }^{53}$ Clinicians must follow strict privacy settings on their digital devices and ensure images are securely stored.

\section{Conclusion}

Due to the highly visual nature of dermatology, imaging has become an essential part of assessing and treating cutaneous diseases. Currently, imaging is used for documenting conditions, monitoring at-risk patients, communication, and academics. Advancements in digital imaging technology, including smaller, more portable devices and overall improved quality of imaging has augmented its utility in dermatology.

Handheld cameras and smartphones that provide high-resolution images have increased clinician's ability to effortlessly capture and document the state of a patient's skin condition or lesions in a multitude of settings. Furthermore, novel, noninvasive imaging devices are currently under investigation for their ability to facilitate diagnosis and management of lesions. The ideal imaging technology would have increased diagnostic accuracy, be time-efficient, reduce unnecessary biopsies, and be accessible to a wide range of patients and physicians, including primary care physicians. It is likely that with advancements and improved standardization of imaging in dermatology, we may be able to better capture and monitor skin conditions over time and achieve better diagnostic accuracy, resulting in fewer biopsies, decreased morbidity and costs, and earlier detection of skin cancer.

\section{References}

1. Witmer WK, Lebovitz PJ. Clinical photography in the dermatology practice. Semin Cutan Med Surg. 2012;31(3):191-199.

2. Thomas L, Tranchand P, Berard F, Secchi T, Colin C, Moulin G. Semiological value of $\mathrm{ABCDE}$ criteria in the diagnosis of cutaneous pigmented tumors. Dermatology. 1998;197(1):11-17.

3. Abbasi NR, Shaw HM, Rigel DS, et al. Early diagnosis of cutaneous melanoma: revisiting the ABCD criteria. JAMA. 2004;292(22):2771-2776.

4. English DR, Del Mar C, Burton RC. Factors influencing the number needed to excise: excision rates of pigmented lesions by general practitioners. Med J Aust. 
2004;180(1):16-19.

5. Accetta P, Accetta J, Kostecki J. The use of digital cameras by US dermatologists. $J$ Am Acad Dermatol. 2013;69(5):837-838.

6. Banky JP, Kelly JW, English DR, Yeatman JM, Dowling JP. Incidence of new and changed nevi and melanomas detected using baseline images and dermoscopy in patients at high risk for melanoma. Arch Dermatol. 2005;141(8):998-1006.

7. Wang SQ, Kopf AW, Koenig K, Polsky D, Nudel K, Bart RS. Detection of melanomas in patients followed up with total cutaneous examinations, total cutaneous photography, and dermoscopy. J Am Acad Dermatol. 2004;50(1):15-20.

8. Dengel LT, Petroni GR, Judge J, et al. Total body photography for skin cancer screening. Int J Dermatol. 2014;54(11):1250-1254.

9. Feit NE, Dusza SW, Marghoob AA. Melanomas detected with the aid of total cutaneous photography. Br J Dermatol. 2004;150(4):706-714.

10. Oliveria SA, Chau D, Christos PJ, Charles CA, Mushlin AI, Halpern AC. Diagnostic accuracy of patients in performing skin self-examination and the impact of photography. Arch Dermatol. 2004;140(1):57-62.

11. Marghoob AA, Usatine RP, Jaimes N. Dermoscopy for the family physician. Am Fam Physician. 2013;88(7):441-450.

12. Vestergaard ME, Macaskill P, Holt PE, Menzies SW. Dermoscopy compared with naked eye examination for the diagnosis of primary melanoma: a meta-analysis of studies performed in a clinical setting. Br J Dermatol. 2008;159(3):669-676.

13. Kittler H, Pehamberger H, Wolff K, Binder M. Diagnostic accuracy of dermoscopy. Lancet Oncol. 2002;3(3):159-165.

14. Bafounta ML, Beauchet A, Aegerter P, Saiag P. Is dermoscopy (epiluminescence microscopy) useful for the diagnosis of melanoma? Results of a meta-analysis using techniques adapted to the evaluation of diagnostic tests. Arch Dermatol. 2001;137(10):1343-1350.

15. Carli P, de Giorgi V, Chiarugi A, et al. Addition of dermoscopy to conventional naked-eye examination in melanoma screening: a randomized study. $\mathrm{J}$ Am Acad Dermatol. 2004;50(5):683-689.

16. Carli P, De Giorgi V, Crocetti E, et al. Improvement of malignant/benign ratio in excised melanocytic lesions in the 'dermoscopy era': a retrospective study 1997-2001. Br J Dermatol. 2004;150(4):687-692.

17. Rosendahl C, Tschandl P, Cameron A, Kittler H. Diagnostic accuracy of dermatoscopy for melanocytic and nonmelanocytic pigmented lesions. J Am Acad Dermatol. 2011;64(6):1068-1073.

18. Menzies SW. Evidence-based dermoscopy. Dermatol Clin. 2013;31(4):521-524.

19. Argenziano G, Mordente I, Ferrara G, Sgambato A, Annese P, Zalaudek I. Dermoscopic monitoring of melanocytic skin lesions: clinical outcome and patient compliance vary according to follow-up protocols. Br J Dermatol. 2008;159(2):331-336.

20. Que SK, Fraga-Braghiroli N, Grant-Kels JM, Rabinovitz HS, Oliviero M, Scope A. Through the looking glass: basics and principles of reflectance confocal microscopy. J Am Acad Dermatol. 2015;73(2):276-284.

21. Pellacani G, Pepe P, Casari A, Longo C. Reflectance confocal microscopy as a second-level examination in skin oncology improves diagnostic accuracy and saves unnecessary excisions: a longitudinal prospective study. $\mathrm{Br} \mathrm{J}$ Dermatol. 2014;171(5):1044-1051.

22. Champin J, Perrot JL, Cinotti E, et al. In vivo reflectance confocal microscopy to optimize the spaghetti technique for defining surgical margins of lentigo maligna. Dermatol Surg. 2014;40(3):247-256.

23. Hibler BP, Cordova M, Wong RJ, Rossi AM. Intraoperative real-time reflectance confocal microscopy for guiding surgical margins of lentigo maligna melanoma. Dermatol Surg. 2015;41(8):980-983.

24. Nadiminti H, Scope A, Marghoob AA, Busam K, Nehal KS. Use of reflectance confocal microscopy to monitor response of lentigo maligna to nonsurgical treatment. Dermatol Surg. 2010;36(2):177-184.

25. Cinotti E, Perrot JL, Campolmi N, et al. The role of in vivo confocal microscopy in the diagnosis of eyelid margin tumors: 47 cases. J Am Acad Dermatol. 2014;71(5):912-918.e2.

26. Debarbieux S, Perrot JL, Erfan N, et al; Groupe d'Imagerie Cutanée Non Invasive de la Société Française de Dermatologie. Reflectance confocal microscopy of mucosal pigmented macules: a review of 56 cases including 10 macular melanomas. $\mathrm{Br} \mathrm{J}$ Dermatol. 2014;170(6):1276-1284.

27. Farnetani F, Scope A, Braun RP, et al. Skin cancer diagnosis with reflectance confocal microscopy: reproducibility of feature recognition and accuracy of diagnosis. JAMA Dermatol. 2015;151(10):1075-1080.

28. Longo C, Farnetani F, Ciardo S, et al. Is confocal microscopy a valuable tool in diagnosing nodular lesions? A study of 140 cases. Br J Dermatol. 2013;169(1):58-67.

29. Gerger A, Koller S, Kern T, et al. Diagnostic applicability of in vivo confo- cal laser scanning microscopy in melanocytic skin tumors. J Invest Dermatol. 2005;124(3):493-498.

30. Ferris LK, Harris RJ. New diagnostic aides for melanoma. Dermatol Clin. 2012;30(3):535-545.

31. Welzel J. Optical coherence tomography in dermatology: a review. Skin Res Technol. 2001;7(1):1-9.

32. Gambichler T, Jaedicke V, Terras S. Optical coherence tomography in dermatology: technical and clinical aspects. Arch Dermatol Res. 2011;303(7):457-473.

33. Ulrich M, von Braunmuehl T, Kurzen H, et al. The sensitivity and specificity of optical coherence tomography for the assisted diagnosis of nonpigmented basal cell carcinoma: an observational study.Br J Dermatol. 2015;173(2):428-435.

34. Masood A, Al-Jumaily AA. Computer aided diagnostic support system for skin cancer: a review of techniques and algorithms. Int J Biomed Imaging. 2013;2013:323268.

35. Koh HK, Miller DR, Geller AC, Clapp RW, Mercer MB, Lew RA. Who discovers melanoma? Patterns from a population-based survey. J Am Acad Dermatol. 1992;26(6):914-919.

36. Maier T, Kulichova D, Schotten K, et al. Accuracy of a smartphone application using fractal image analysis of pigmented moles compared to clinical diagnosis and histological result. J Eur Acad Dermatol Venereol. 2015;29(4):663-667.

37. Nijhawan RI, Lee EH, Nehal KS. Biopsy site selfies--a quality improvement pilot study to assist with correct surgical site identification. Dermatol Surg. 2015;41(4):499-504.

38. Moncrieff M, Cotton S, Claridge E, Hall P. Spectrophotometric intracutaneous analysis: a new technique for imaging pigmented skin lesions. $\mathrm{Br} J$ Dermatol. 2002;146(3):448-457.

39. Haniffa MA, Lloyd JJ, Lawrence CM. The use of a spectrophotometric intracutaneous analysis device in the real-time diagnosis of melanoma in the setting of a melanoma screening clinic. Br J Dermatol. 2007;156(6):1350-1352.

40. Glud M, Gniadecki R, Drzewiecki KT. Spectrophotometric intracutaneous analysis versus dermoscopy for the diagnosis of pigmented skin lesions: prospective, double-blind study in a secondary reference centre. Melanoma Res. 2009;19(3): 176-179.

41. Wilson EC, Emery JD, Kinmonth AL, et al. The cost-effectiveness of a novel SIAscopic diagnostic aid for the management of pigmented skin lesions in primary care: a decision-analytic model. Value Health. 2013;16(2):356-366.

42. Gutkowicz-Krusin D, Elbaum M, Jacobs A, et al. Precision of automatic measurements of pigmented skin lesion parameters with a MelaFind(TM) multispectral digital dermoscope. Melanoma Res. 2000;10(6):563-570.

43. Friedman RJ, Gutkowicz-Krusin D, Farber MJ, et al. The diagnostic performance of expert dermoscopists vs a computer-vision system on small-diameter melanomas. Arch Dermatol. 2008;144(4):476-482.

44. Monheit G, Cognetta AB, Ferris L, et al. The performance of MelaFind: a prospective multicenter study. Arch Dermatol. 2011;147(2):188-194.

45. Wells R, Gutkowicz-Krusin D, Veledar E, Toledano A, Chen SC. Comparison of diagnostic and management sensitivity to melanoma between dermatologists and MelaFind: a pilot study. Arch Dermatol. 2012;148(9):1083-1084.

46. Cukras AR. On the comparison of diagnosis and management of melanoma between dermatologists and MelaFind. JAMA Dermatol. 2013;149(5):622-623.

47. Rigel DS, Roy M, Yoo J, Cockerell CJ, Robinson JK, White R. Impact of guidance from a computer-aided multispectral digital skin lesion analysis device on decision to biopsy lesions clinically suggestive of melanoma. Arch Dermatol. 2012;148(4):541-543.

48. Hauschild A, Chen SC, Weichenthal M, et al. To excise or not: impact of MelaFind on German dermatologists' decisions to biopsy atypical lesions. J Dtsch Dermatol Ges. 2014;12(7):606-614.

49. Shoo BA, Sagebiel RW, Kashani-Sabet M. Discordance in the histopathologic diagnosis of melanoma at a melanoma referral center. $J$ Am Acad Dermatol. 2010;62(5):751-756.

50. Lim L, Nichols B, Migden MR, et al. Clinical study of noninvasive in vivo melanoma and nonmelanoma skin cancers using multimodal spectral diagnosis. J Biomed Opt. 2014;19(11):117003.

51. Quigley EA, Tokay BA, Jewell ST, Marchetti MA, Halpern AC. Technology and technique standards for camera-acquired digital dermatologic images: a systematic review. JAMA Dermatol. 2015;151(8):883-890.

52. Hsieh C, Yun D, Bhatia AC, Hsu JT, Ruiz de Luzuriaga AM. Patient perception on the usage of smartphones for medical photography and for reference in dermatology. Dermatol Surg. 2015;41(1):149-154.

53. Anyanwu CO, Lipoff JB. Smartphones, photography, and security in dermatology. $J$ Am Acad Dermatol. 2015;72(1):193-195. 